\title{
Volatiles Loss from water bearing regolith simulant at Lunar Environments
}

\author{
J. Kleinhenz ${ }^{1}$, J. Smith ${ }^{2}$, T. Roush ${ }^{3}$, A. Colaprete ${ }^{3}$, K. Zacny ${ }^{4}$, G. Paulsen ${ }^{4}$, A. Wang ${ }^{4}$, \\ and A. $\mathrm{Paz}^{5}$ \\ ${ }^{1}$ NASA Glenn Research Center, Cleveland, Ohio, 44135 \\ ${ }^{2}$ NASA Kennedy Space Center, Florida 32899 \\ ${ }^{3}$ NASA Ames Research Center, Mountain View, CA 94035 \\ ${ }^{4}$ Honeybee Robotics Spacecraft Mechanisms Corporation, Pasadena, Calf. 91103 \\ ${ }^{5}$ NASA Johnson Space Center, Houston, TX, 77058
}

\begin{abstract}
In-Situ Resource Utilization (ISRU) enables future planetary exploration by using local resources to acquire mission consumables. Water-bearing regolith has been identified on the moon in the permanently shadowed craters. Missions designed to retrieve these resources will require testing in relevant environments.
\end{abstract}

The Planetary Surface Simulation Facility (otherwise known as "VF-13") at the NASA Glenn Research Center can create these relevant environments for ground based testing. This dirty thermal vacuum chamber is $3.6 \mathrm{~m}$ tall, $1.5 \mathrm{~m}$ in diameter, and can achieve pressures on the order of $10^{-6}$ Torr. The internal wall of the chamber and the soil bin are separately temperature controlled using liquid nitrogen.

For the past four years, the chamber has been used by NASA's Resource Prospector to characterize volatiles loss during regolith sampling operations. Observations from 43 samples suggest agitating the sample during delivery has a significant impact on the volatiles loss. Calculated mass loss rates are consistent for similar size samples. However, the variations in moisture loss do not clearly correlate with measured conditions. Continued testing will examine the impacts of the mechanical sample delivery process.

\section{INTRODUCTION}

In Situ Resource Utilization (ISRU) enables future planetary exploration by using local resources to acquire mission consumables, which potentially reduces mission cost and risk. Water-bearing regolith has been identified on several planetary bodies including the moon, in the permanently shadowed craters, on Mars, and potentially on other moons and asteroids. Missions designed to retrieve these resources will require testing in relevant environments. The Planetary Surface Simulation Facility (otherwise known as "VF-13") at the NASA Glenn Research Center can create these relevant environments for ground based testing. For the last 5 years (Kleinhenz 
(2015), Kleinhenz (2014)), this facility has supported a potential lunar polar mission called Resource Prospector.

The goal of Resource Prospector (RP) is to characterize the nature and distribution of water in the lunar polar subsurface in order to inform ISRU strategy (Andrews (2014)). To accomplish this, the RP payload is a rover mounted system including: a drill, a neutron spectrometer (the Neutron Spectrometer Subsystem (NSS)), a nearinfrared spectrometer (the Near InfraRed Volatiles Spectrometer Subsystem (NIRVSS)), a reactor (the Oxygen and Volatile Extraction Node (OVEN)), and a gas chromatograph/mass spectrometer (The Lunar Advanced Volatiles Analysis (LAVA) Subsystem). The two spectrometer sub-systems (NSS and NIRVSS) are intended to evaluate the near surface material to identify water 'hot spots'. The drill subsystem is then used to obtain a subsurface sample at those locations for more detailed evaluation in the OVEN and LAVA subsystems. Thus far, three of the RP subsystems have been integrated into the thermal vacuum chamber for testing: Drill, NIRVSS, and OVEN.

The goal of the RP integrated thermal vacuum test program is to understand the subsurface sampling process and its impact on volatile retention. The intent is to relate the volatile content within the surface to the amount observed and measured by the RP instrumentation. The act of extracting the regolith sample, and the interaction between the regolith sample and the hardware, will cause some amount of volatiles release. This impact must be understood in order to properly interpret the results. The thermal vacuum tests will be used to bound the potential water loss during the sampling process in order to define uncertainties in the measured sample. This information is also used to improve theoretical predictions. Additionally, the integrated testing continues to inform hardware design and mission concept of operations.

A total of 10 test entries examining volatile release were performed over the course of 4 years. An additional 2 tests during a prior year focused on hardware performance only, and did not include volatile sampling. A 'test' is defined as an entry into the thermal vacuum chamber with a fresh soil bin. Each soil bin supports 4 to 6 drill holes and 5 volatile retention samples. A total of 43 viable soil samples have been recovered to date.

\section{RESOURCE PROSPECTOR HARDWARE}

Figure 1 shows photographs of the RP hardware configurations for the VF-13 test programs. Each of these hardware components will be detailed in the following sections. In the image at left, the drill is delivering the sample to the OVEN during the 2017 test program. The crucible can be seen extended out of the OVEN system with the drill funnel positioned above. The image at right shows the Sample Crucible Mechanisms (SCMs) which were frequently used in place of the OVEN to collect samples. This image was taken during the 2016 test program so there are slight differences in the drill and NIRVSS hardware. 

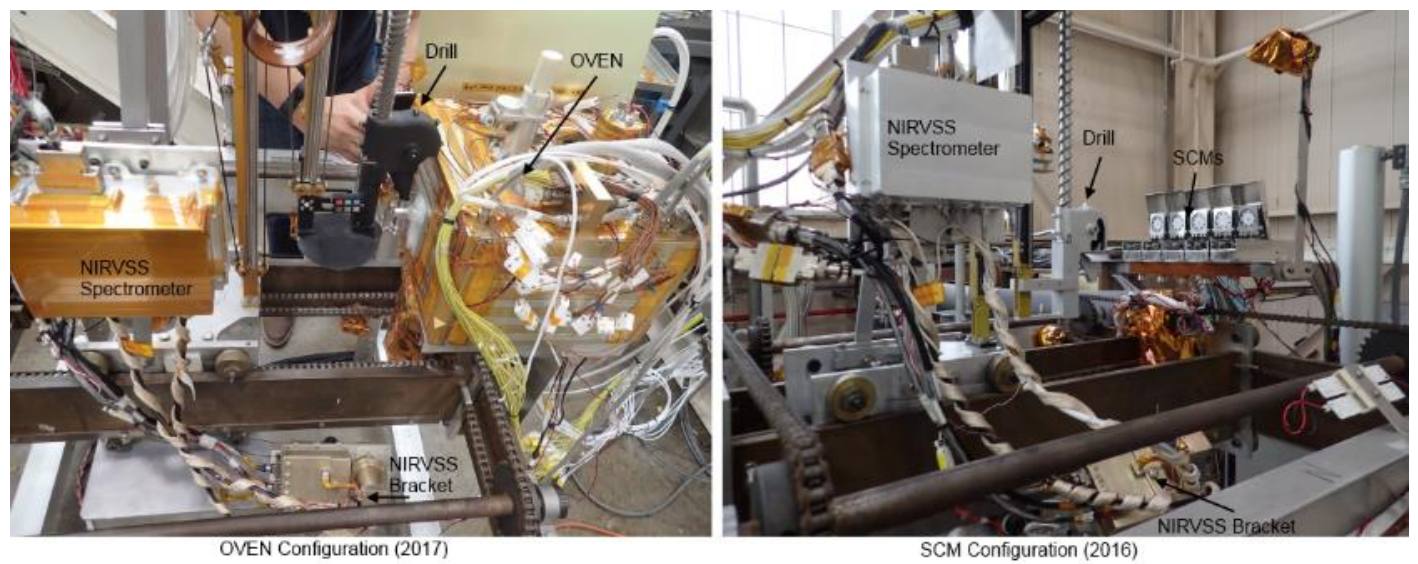

Figure 1: The two hardware configurations used in the RP thermal vaccum test program. At left, the OVEN subsystem was used for sample collection and storage, while at right the SCMs were used.

Drill. The Resource Prospector Drill is based on the Icebreaker drill developed for penetrating ice and ice cemented ground for Mars applications (Zacny et al., 2013). The RP drill does not need to meet planetary protection requirements that are required for Mars Special Regions (Cat IVc) and also does not require the same level of autonomy. The other differences include deployment platforms: the Icebreaker mission is lander based and requires 3 DoF Robotic Arm deployments whereas Resource Prospector is rover based and as such requires 1 DoF deployment using a Deployment Stage.
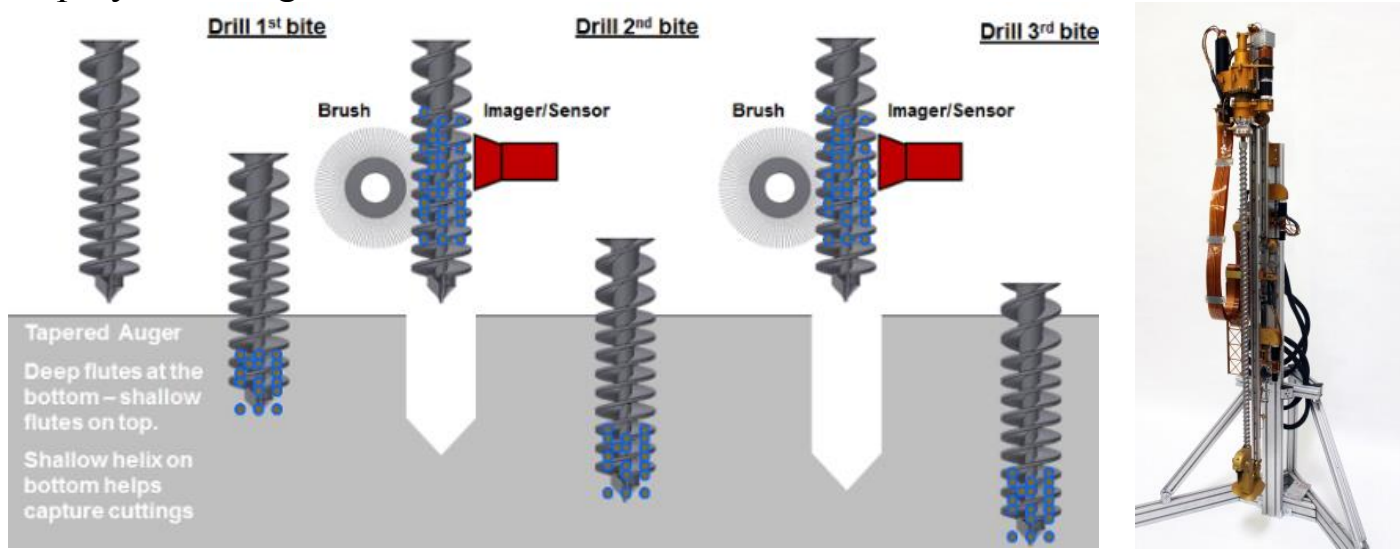

Figure 2. The 'bite' sampling sequence used to retrieve soil samples from depth is shown at left. At right, an image of the TRL6 RP drill.

The drill supports a $100 \mathrm{~cm}$ long, $2.5 \mathrm{~cm}$ diameter auger which is designed to capture a sample on the lowest $10 \mathrm{~cm}$ section of the auger. This section has flutes which are deep and placed at low pitch in a geometry ideal for retaining of granular material. The geometry of the upper section flutes are shallow and with higher pitch which is best suited for efficient cuttings transport. The approach to drilling and sample capture is 'bite' sampling (or peck drilling in machine shop terminology), which 
involves progressive drilling and sample capture in $10 \mathrm{~cm}$ deep sections (Figure 2). An advantage to this approach is that the depth stratigraphy of the hole is preserved (per $10 \mathrm{~cm}$ interval). Since the auger does not have to convey the full depth of material to the surface at once, the auger power and the risk of getting stuck in the hole is substantially reduced. A passive brush is used to clear the sample from the auger for instrument analysis; either depositing the sample on the surface for the NIRVSS or into a sample crucible for OVEN and LAVA processing.

The drill has undergone several re-design cycles to reduce its mass and increase its Technology Readiness level (TRL) (Paulsen et al., 2016). The current TRL of the drill is TRL6.

NIRVSS. The NIRVSS engineering test unit (ETU) is described in detail by Roush (2016) and consists of two separate components shown in Figure 3. The spectrometer box contains two, fiber-optic fed, near-infrared spectrometers. The bracket assembly (BA) contains connections for the fiber optic cables, an infrared illumination source (aka lamp), a drill observation camera (DOC), light emitting diodes (LEDs), and a Longwavelength Calibration Sensor (LCS) to document surface and subsurface temperature. Since Roush (2016), the NIRVSS ETU has been updated (ETU+) to include some additional components.

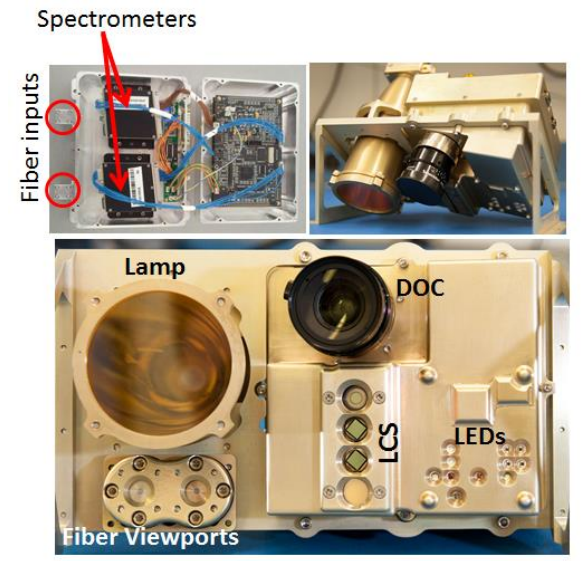

Figure 3. The Near InfraRed Volatiles Spectrometer Subsystem (NIRVSS).

The NIRVSS spectrometers remain the same, covering $\sim 1600-2400 \mathrm{~nm}$, at $\sim 9 \mathrm{~nm}$ sampling, and $\sim 2300-3400 \mathrm{~nm}$ at $\sim 12 \mathrm{~nm}$ sampling. On the NIRVSS BA, the lamp and DOC are unchanged from the description in Roush (2016). In VF-13, the DOC with an $\mathrm{f} / 2.5$ lens and operational distances from the soil surface provide a spatial resolution of about $\sim 0.125 \mathrm{~mm} /$ pixel. In the NIRVSS ETU+ the number of LEDs is increased (adding 540, 640, 940 and $1050 \mathrm{~nm}$ ) and the LCS has a reduced field of view (from 60 to $\sim 36$ degrees) and the wavelengths are now 7.9, $10.6,14$, and $25 \mu \mathrm{m}$; selected to provide sensitivity to the $200-350 \mathrm{~K}$ range.

OVEN. The RP OVEN subsystem (Figure 4) is designed to capture regolith samples dispensed from the drill, seal the sample in a crucible, and then heat those samples to release volatiles. The OVEN is designed to reuse the $12 \mathrm{~cm}^{3}$ crucibles, so a robotic arm mechanism accommodates sample acquisition, heating, and disposal. Figure 1 shows the crucible beneath the drill sample capture assembly to receive the sample. 


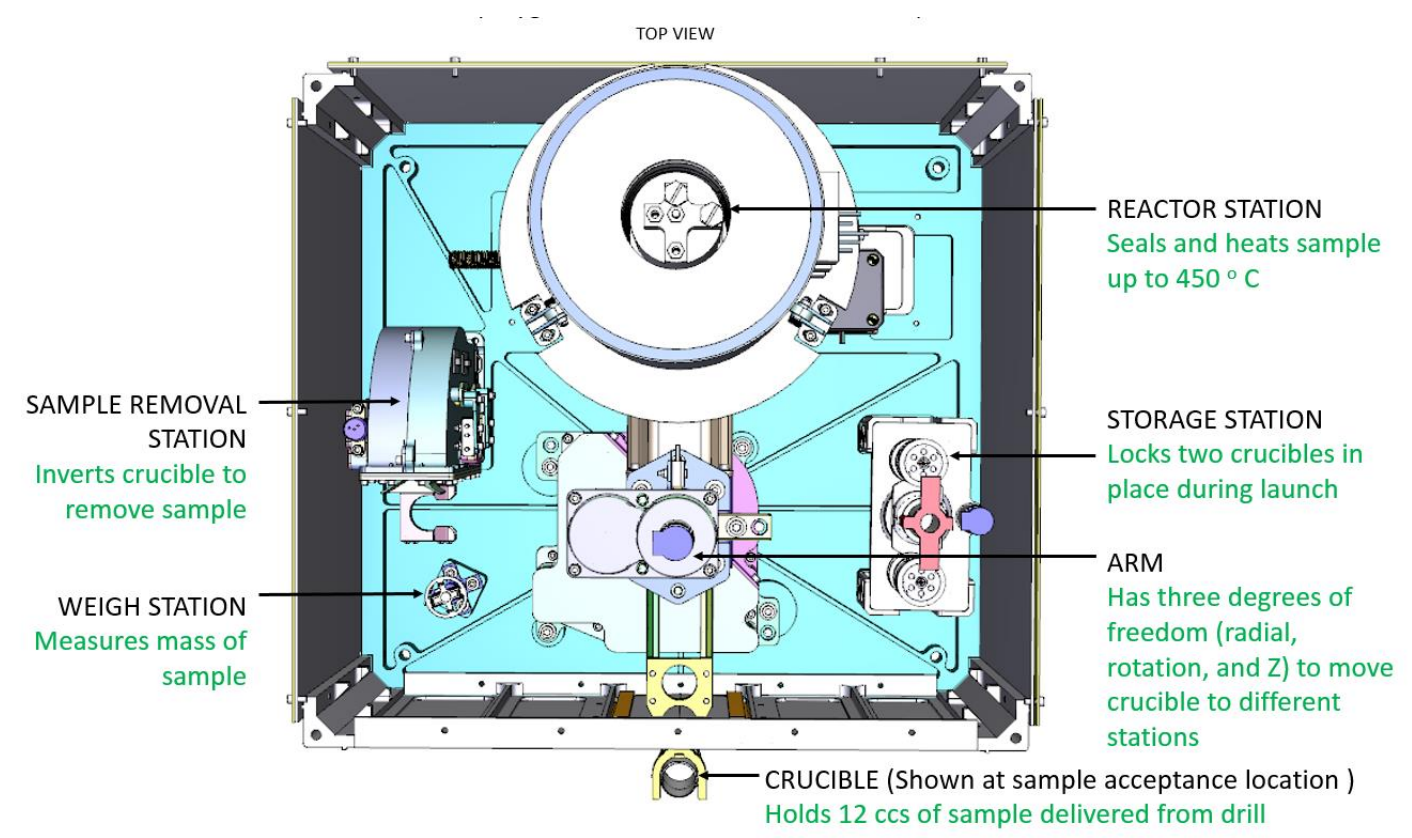

Figure 4. Oxygen and Volatile Extraction Node (OVEN).

A modified version of the RP OVEN was used for testing in VF-13. The samples were not heated in the sealed vessel, since the volatiles analysis subsystem (LAVA) was not available. Instead, the crucible was sealed and stored at the reactor station for post-test analysis. A removable cap containing two axial o-rings was installed in place of the heated seal mechanism (figure 5). Because the OVEN accommodated one crucible at time, vacuum conditions were interrupted to retrieve each sample.

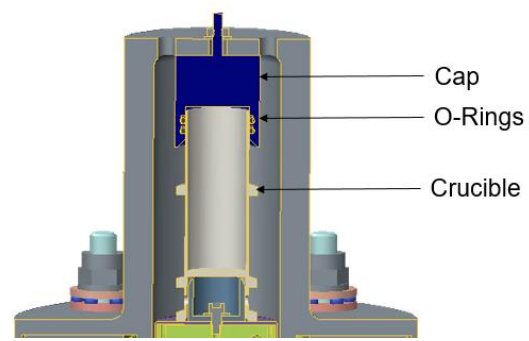

Figure 5. A cross sectional view of the modified OVEN crucible seal system used for the thermal vacuum test program.

Sample Capture Mechanisms. A set of five specialized sample capture mechanisms (SCMs) can be used in place of the OVEN subsystem for thermal vacuum chamber testing. Designed and built specifically for the integrated test program, these units allow acquisition of multiple soil samples for each vacuum test. A SCM (Figure 6) consists of a removable sample crucible and a remotely actuated sealing mechanism.

The $18 \mathrm{~mL}$ (1.9 $\mathrm{cm}$ diameter) crucible was designed to hold a projected $10 \mathrm{~mL}$ of soil. The diameter of the crucible is matched to that of the drill's funnel to minimize soil spillage. A teflon seal contained in the crucible lid is driven into a the crucible's knife-edge flange using an remotely actuated stepper motor. A four bar linkage ensures that the lid assembly is parallel to the crucible in the clamping position so that the force is evenly distributed. The crucible vent screw, shown in figure 6 was eliminated in 2017 to improve seal integrity. 
After each test is complete, the sealed crucibles are removed for laboratory analysis. The same sample analysis method is used for both the SCM and OVEN crucibles. The moisture content of each sample is determined using standard method ASTM (2005); which involves measuring the mass change upon bake out at $110^{\circ} \mathrm{C}$. The moisture content of these samples is compared to the moisture level of the soil bin to determine the loss. Since the moisture content of the soil bin cannot be measured at vacuum, a post test evaluation is used to evaluate the moisture content of the bin.

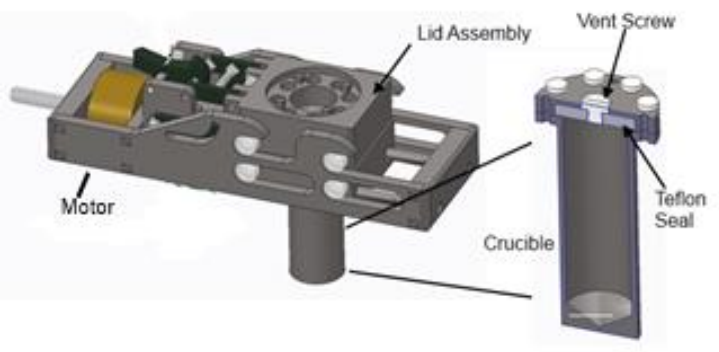

Figure 6. The Sample Crucible Mechanism (SCM) hardware.

\section{THERMAL VACUUM TEST ENVIRONMENT}

The thermal vacuum facility, called VF-13, is a $3.6 \mathrm{~m}$ tall vertical, cylindrical chamber with an internal volume of $6.35 \mathrm{~m}^{3}$ (figure 7). The bulk of the volume is within the removable $2.52 \mathrm{~m}$ tall by $1.5 \mathrm{~m}$ diameter lid, while the fixed base of VF-13 is $1.08 \mathrm{~m}$ deep and accommodates all the electrical, mechanical, and gas feedthroughs. A removable cold wall can be placed inside the lid (inner diameter with cold wall is $1.35 \mathrm{~m}$ ) and is composed of two semi-circular sections that can be separately controlled to mimic the temperature gradients on the lunar surface. An additional cold shell is clamped around the soil bin, which sits in the fixed base of the chamber. The cold walls and soil bin can be independently temperature controlled from atmospheric temperature to $\sim 90 \mathrm{~K}$ using Liquid Nitrogen, which is amply supplied from a 55,000 gallon dewar. During testing with simulant, chamber pressures are typically $5 \mathrm{e}^{-6}$ Torr. A residual gas analyzer (RGA) is mounted

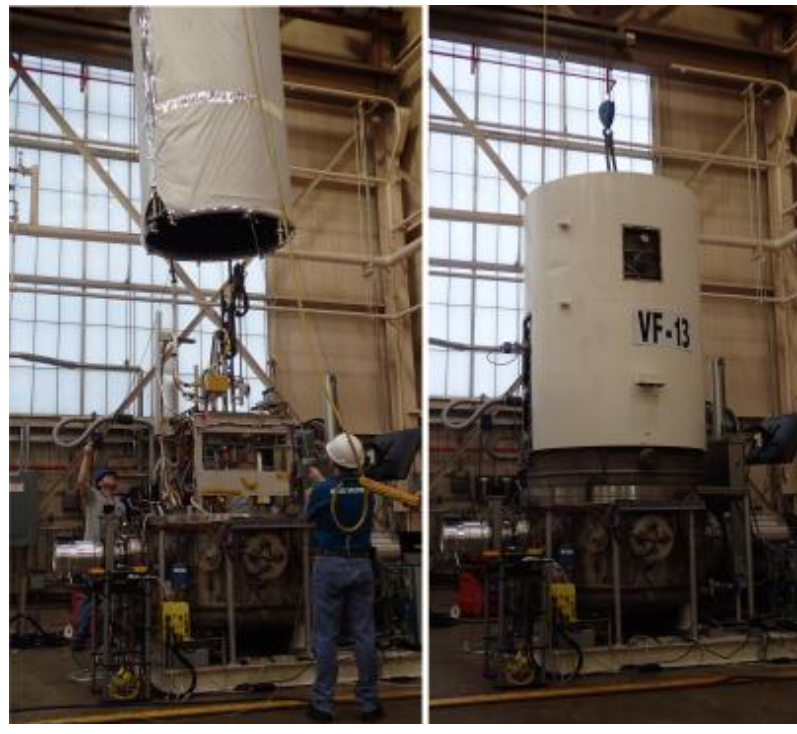

Figure 7. The VF-13 thermal vacuum facility at NASA GRC. At left, the RP hardware is visible as the cold wall is installed. At right, the $3.6 \mathrm{~m}$ tall chamber is sealed. 
to the chamber to examine gas composition during testing. An internal Mass Spectrometer was used during one test program.

The regolith simulant is contained in a cylindrical aluminum bin $1.2 \mathrm{~m}$ tall with an inner diameter of $0.278 \mathrm{~m}$. The bin height accommodates the $1 \mathrm{~m}$ long drill, and the diameter permits multiple drill holes while keeping heat transfer time (chill down) to a reasonable time frame. Three ports at various heights along the soil bin accommodate thermocouple probes. Each probe can support five type-T thermocouples which are embedded in the soil at different radial positions, for a total of 15 thermocouples. For RP testing, the bin is filled with $\sim 100 \mathrm{~kg}$ of water-doped LHT-3M simulant and vibrationally compacted in 5 layers (Kleinhenz (2013)).

The drill and NIRVSS are mounted to a trolley that can be moved in two dimensions while at thermal vacuum conditions using remotely actuated stepper motors and chain drives. This gives access to the entire surface of the soil bed so that multiple drill holes can be accomplished in a single test. The trolley also allows access to the OVEN/SCMs which are stationary-mounted to the trolley frame.

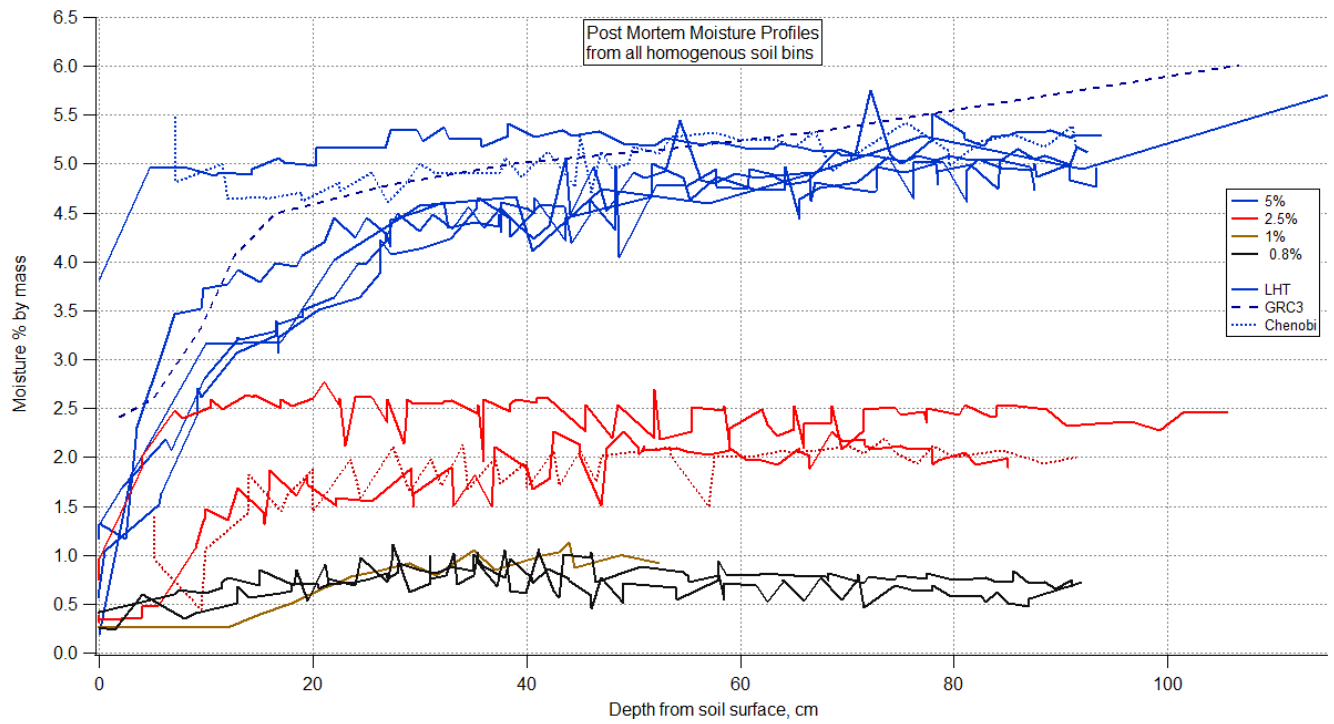

Figure 8. The moisture profile of various VF-13 soil bins based on post-test sampling. Colors indicate the target pre-test moisture condition, while line type indicates simulant type.

\section{TEST OPERATIONS}

Chamber Conditioning: It typically takes 3-4 days to condition the chamber for a test. The first day ( $\sim 6$ hours) is the rough pump period which must be closely monitored. Off gassing of the soil during pump down can cause significant soil disruptions (Kleinhenz (2014)), especially in the 1 to 10 Torr range. The pump rate is reduced with $\mathrm{GN}_{2}$ bleeds to mitigate this. The chilling of the soil bed to a target of $-100^{\circ} \mathrm{C}$ is the most time consuming process. A higher moisture content soil bed will reach temperature faster than a dry bed. Pre-chilling of the bed prior to and/or during 
pump down expedites this. The cold wall is active once pressures reach the milliTorr scale to temperature condition the hardware.

Vacuum exposure will cause desiccation of the soil bed. Figure 8 shows the post-test moisture profile for a variety of soil beds used in VF13. The majority of desiccation takes place in the top $20-30 \mathrm{~cm}$ of the bed. While there can be some moisture gradient beyond this, particularly with the wetter condition, it is greatly reduced. In most cases the desiccation is restricted to $\sim 0.5 \mathrm{wt} \%$ below $30 \mathrm{~cm}$. These profiles represent a thawed condition, but a few early tests were performed on a frozen bin immediately after breaking vacuum (Kleinhenz (2014)). In these cases, the desiccated layer was shallower but drier. So some redistribution of moisture occurs during thawing process, but the bulk of it is restricted to the top $30 \mathrm{~cm}$. Therefore, all the soil samples for RP were taken from $30 \mathrm{~cm}$ or below.

Drilling: Each soil bin will support 4-5 holes, depending on hardware configuration. Using the bite approach, the hole is formed in $10 \mathrm{~cm}$ increments. The first 3 bites ( $0 \mathrm{~cm}$ to $30 \mathrm{~cm}$ depth) are deposited on the soil surface for inspection by the NIRVSS instrument. The bite between 30 and $40 \mathrm{~cm}$ is transferred to the sample crucible in either the SCM or OVEN for post-test measurement. This involves fully extracting the drill from the hole and raising the foot. However for some tests, the drill is repositioned over the same hole to continue drilling.

Variables: Over the course of the multiyear program a variety of variables have been used to study various concept of operations and hardware performance.

Pressure: Chamber pressure was typically kept as low as possible. At least one pump was active during test operations to achieve this. Average test pressure was $4 \mathrm{e}^{-6}$ Torr with a median of $3 \times 10^{-6}$ Torr.

Cold Wall Temperature: The cold wall temperature governs the hardware temperature (drill, OVEN, SCMs, etc) via radiation. During the test program, the cold wall was typically controlled to $-50^{\circ} \mathrm{C}$ in order to mimic surface temperature condition in a sunlit region at the lunar poles. However several tests were performed with the cold wall at $-170^{\circ} \mathrm{C}$ to simulate a permanently shadowed region at the lunar poles.

Soil Bin Temperature: The goal was to get the average soil temperature to at least $-100^{\circ} \mathrm{C}$ prior to testing. Drier soil bins were generally warmer than those with a higher moisture content. The temperature range for the testing was $-80^{\circ} \mathrm{C}$ to $-160^{\circ} \mathrm{C}$, all but three were below the $-100^{\circ} \mathrm{C}$ target.

Soil Bin Moisture Content: The soil bins all consisted of LHT-3M lunar soil simulant doped with water. The highest moisture content was $5 \mathrm{wt} \%$, as identified at the Lunar poles using orbital assets (Colaprete (2010)). Four of the ten tests were performed with a homogenous moisture content of $5 \mathrm{wt} \%$, two tests with $2.5 \mathrm{wt} \%$, and two 0.8 $\mathrm{wt} \%$. Stratified soil beds were used for two tests with alternating layers of $5 \mathrm{wt} \%$ and room condition $(0.3 \mathrm{wt} \%$ to $0.5 \mathrm{wt} \%)$ soil. The drilled samples for the stratified bin were taken from a $5 \mathrm{wt} \%$ layer.

Crucible Temperature: For the majority of tests, the sample crucibles were left uncontrolled, typically reaching $-20^{\circ} \mathrm{C}$, with a $-50^{\circ} \mathrm{C}$ cold wall set point. Seven crucibles were heated to $+10^{\circ} \mathrm{C}$ to mimic a 'storage' temperature for the crucible, as 
influenced by the environment inside the rover. The 3 OVEN crucibles were stored in a $+20^{\circ} \mathrm{C}$ environment and only exposed to cold wall a few minutes before accepting the sample. There was no direct measurement of these OVEN crucible temperatures.

Exposure Time: After initial conditioning, the soil samples experienced the greatest exposure to the environment as they were being delivered to the crucible. During this time they were exposed to hardware temperatures including the drill, brush, funnel, and the crucible. The samples were also agitated during delivery which exposes more surface area. It took about 5 min to brush the sample into the crucible and seal it in. In several tests the sealing process was intentionally delayed $3 \mathrm{~min}$ to examine a potential con-ops scenario.

Sample Size: For the SCMs the target sample mass was $15 \mathrm{~g}$. However, hardware configuration changes resulted in a range of sample masses. The average size of the 43 samples was $12 \mathrm{~g}$ with a median of $14 \mathrm{~g}$.

\section{RESULTS AND DISCUSSION}

The following discussion highlights some trends and overall observations seen thus far. The goal of this analysis was to characterize consistent volatile loss caused by the sampling operations, and identify the primary factors driving that loss. This information will be used to bound potential losses that may occur on the lunar mission. Figure 9 shows an overview of the results, and highlights some of the complexities in this type of characterization. The percent of water loss is plotted against the moisture content of the soil bed at sample depth, as determined by the post-test soil bed analysis (eg Figure 8). The size of the symbols are proportional to the mass of the sample recovered and the color indicates the temperature of the soil bed. The sampling process had a greater impact (greater water loss) when the soil bed was drier. The samples from a 4.5 wt $\%$ bin averaged $\quad 30 \%$ water loss, whereas samples from a $0.8 \mathrm{wt} \%$ bed resulted in losses around $80 \%$. However, the range of the water loss at each condition is significant.

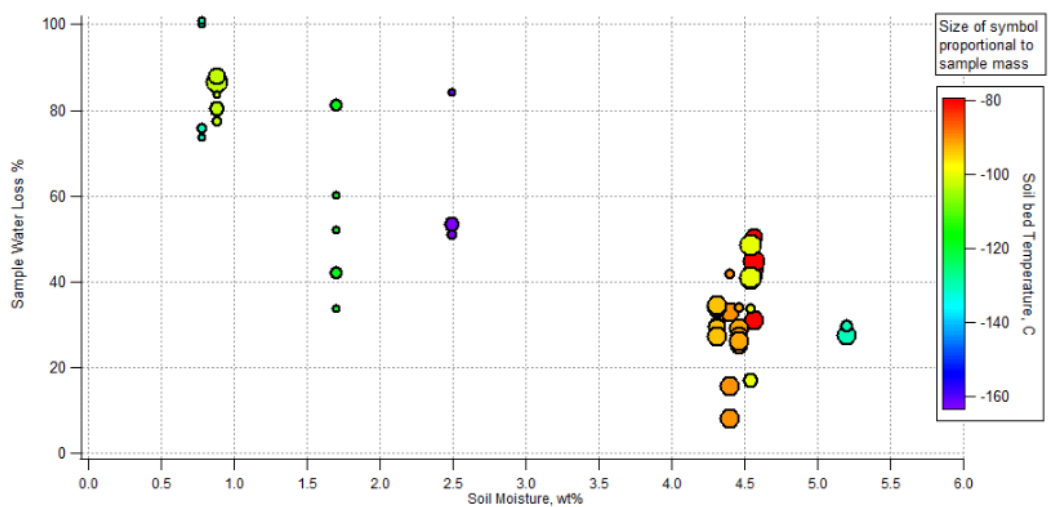

Figure 9. The percent water loss is plotted against soil bin moisture content for all the samples.

To examine this further, mass loss rate of water is plotted against soil bin moisture in Figure 10A. The mass loss rate is the mass of water lost from the sample divided by the exposure time of the sample out of the hole until it is sealed in the crucible. The soil bin's post-test moisture content at sampling depth was used to represent the maximum potential water in the sample. The color in figure 10 indicates the sample 
water loss as percent, which echoes the observation from figure 9 that a wetter soil bed resulted in less water loss from the sample. However, the data in Figure 10A is clustered by symbol size which is proportional to the mass of the collected sample. This mass dependence is very clear in Figure 10B. So it stands to reason that if the mass loss rate is consistent for samples of similar size, samples with a higher starting moisture will have a lower percentage loss.

Mass loss rate should be representative of the sublimation rate, where the scatter in the data should be due to temperature variations per test (since pressures are similar). However, the sample is exposed to several temperatures during the course of acquisition and transfer. So the question is: which temperature is driving the majority of volatiles sublimation? In other words, what part of the transfer/acquisition process is driving water loss? Figure 11 shows the mass loss rate plotted against the four measured temperatures which would directly impact the sample. The plot in (a) shows the temperature of the soil bed, which is the starting temperature of the sample. There are three factors that should be
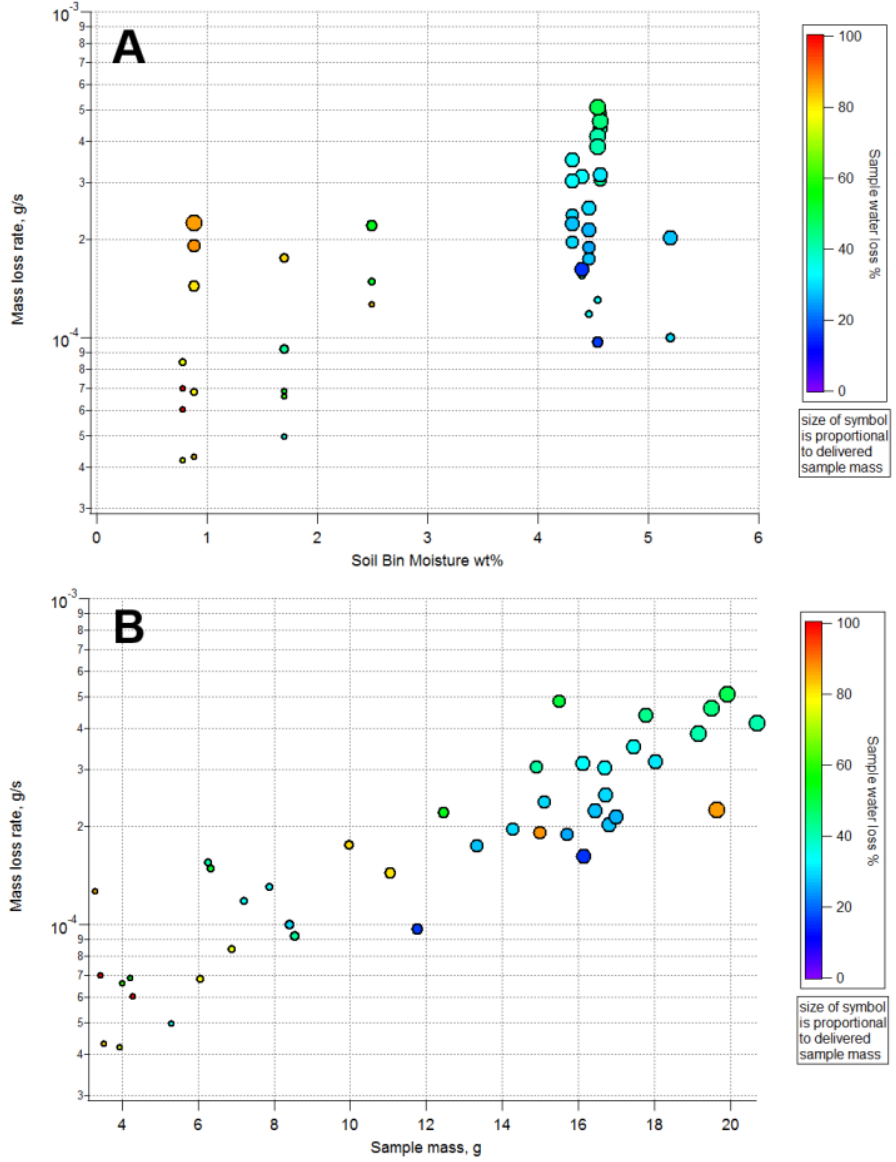

Figure 10. Mass loss rate of the collected samples versus (A) soil bed water content and (B) sample wet mass. The color indicates the percent water loss of each sample, while symbol size is proportional to sample wet mass.

noted when considering the soil bed temperature impact. First, Figure 8 illustrated that soil at the sampling depth $(30$ to $40 \mathrm{~cm}$ ) would not be exposed to loss until the soil layer above it is reduced. So the soil at this depth, and thus at this temperature, would only be exposed during a portion of the $20 \mathrm{~min}$ it takes to drill to $30 \mathrm{~cm}$ using the bite approach. Even then the exposure is not direct. Second, the post-test moisture content of the bin was used in the analysis, so total moisture loss from a warmer bin would be factored in. Third, there are 3 points in Figure $11 \mathrm{~A}$ at $-160^{\circ} \mathrm{C}$ that are outliers. These points represent samples delivered to the OVEN, whereas all other samples were delivered to the SCMs. This difference suggests that other aspects of 
the delivery process have a larger impact on the loss. Even considering all these things, there is no strong trend in Figure 11a, especially when considering that data here is clustered according to sample mass (symbol size). However, the soil bed temperature will impact the temperature of the drill bit, which is shown in Figure 11C. The bit temperature averages $40^{\circ} \mathrm{C}$ higher than the soil temperature. It appears the higher bit temperatures tend to have a higher loss rate. However, the lowest bit temperatures also happen to have lower sample mass, which from Figure 10B has a clear impact on loss rate. Figures 11B and 11D show the cold wall (environmental) temperature and crucible temperature respectively. Neither appear to have a strong correlation with mass loss. This is particularly interesting since even the heated crucibles $\left(\geq 10^{\circ} \mathrm{C}\right)$ do not show significantly higher losses neither in terms of percent water loss nor loss rate. So in terms of measured temperatures, there is no clear driver.

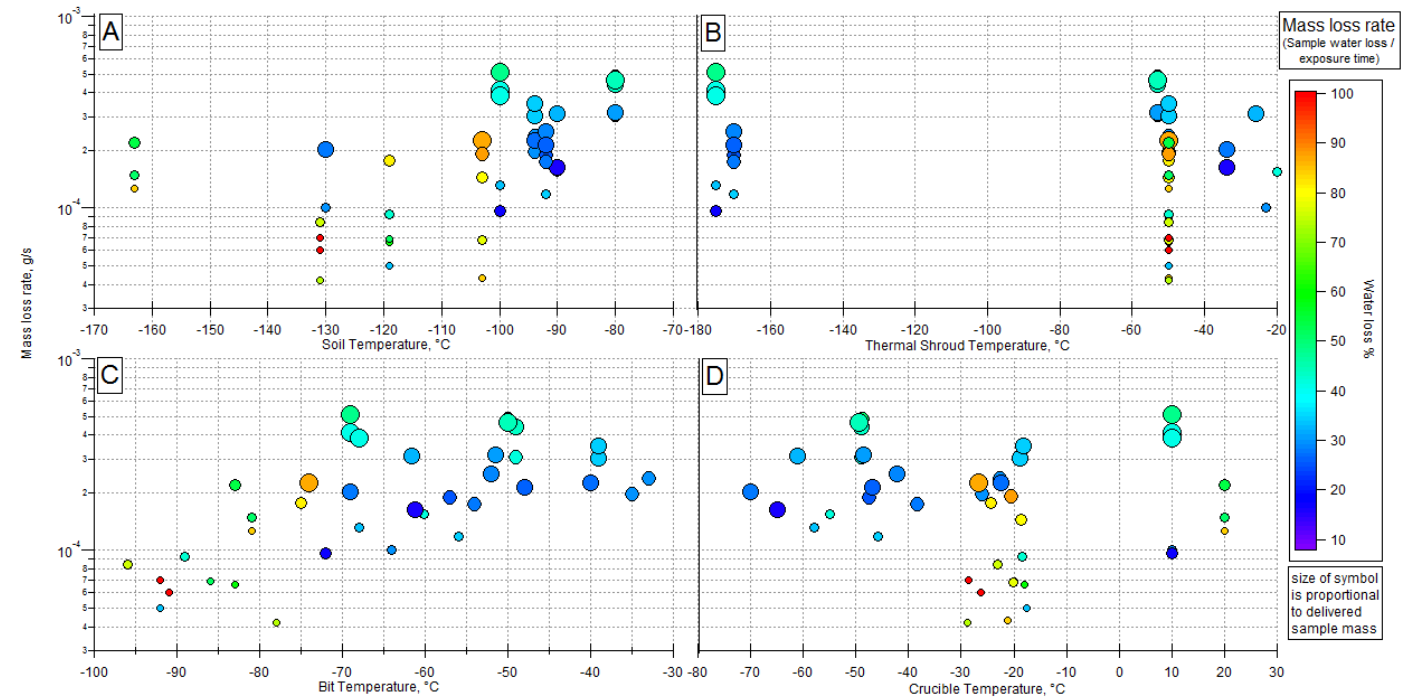

Figure 11. Mass lost rate of the samples plotted against the measured exposure temperatures. During acquisition and delivery the sample is exposed to temperature of (A) soil bin, (B) cold wall, (C) drill bit, and (D) crucible.

Exposure time is another factor in volatiles loss, which is shown in Figure 12 with respect to the water loss. To help identify when the majority of loss is occurring, this was approached three different ways. In Figure 12A, the exposure time starts when drill is at sampling depth and includes the entire retraction and delivery process. The time in (B) starts when the bit is out of the hole (when the sample is fully exposed to the environment) and ends when the crucible is sealed. The final plot only covers the sample delivery process, from when the brushing process begins until the crucible is sealed. There is no clear trend here to help indicate which segment of time may be driving loss. If anything (C) seems to suggest the counterintuitive answer that a longer exposure results in less water loss. For the mass loss rates reported here, the time in (B) was used.

The Residual Gas Analyzer (RGA) instrument was used during the entire drilling operations, and can qualitatively identify peak times of water release. These results 
are shown in Figure 13, which is an overview of a single test. The plot in (B) is the drill data, where the right axis corresponds to drill depth. The peaks indicate the bit is at depth. The left axis indicates bit temperature which declines over the course of the test as the bit spends more time in contact with the colder soil bed. When the bit is out of the hole, it appears to retain its temperature well. The RGA results are shown in (C). The blue line indicates mass number 18, water. The large peaks of the water signal occur during transfer of the sample to the crucibles (indicated by the gray bands). While there are some peaks during drilling, they are much lower magnitude. These peaks are corroborated by the total chamber pressure trace in (D). This seems to be a clear indication that significant water loss is occurring when the sample is being brushed off the drill bit into the crucible. Since the crucible temperature Figure $11 \mathrm{D}$ did not show a strong correlation to loss, it appears it is the agitation of the sample, the act of brushing of the auger and the material falling into the crucible that is spurring the release.

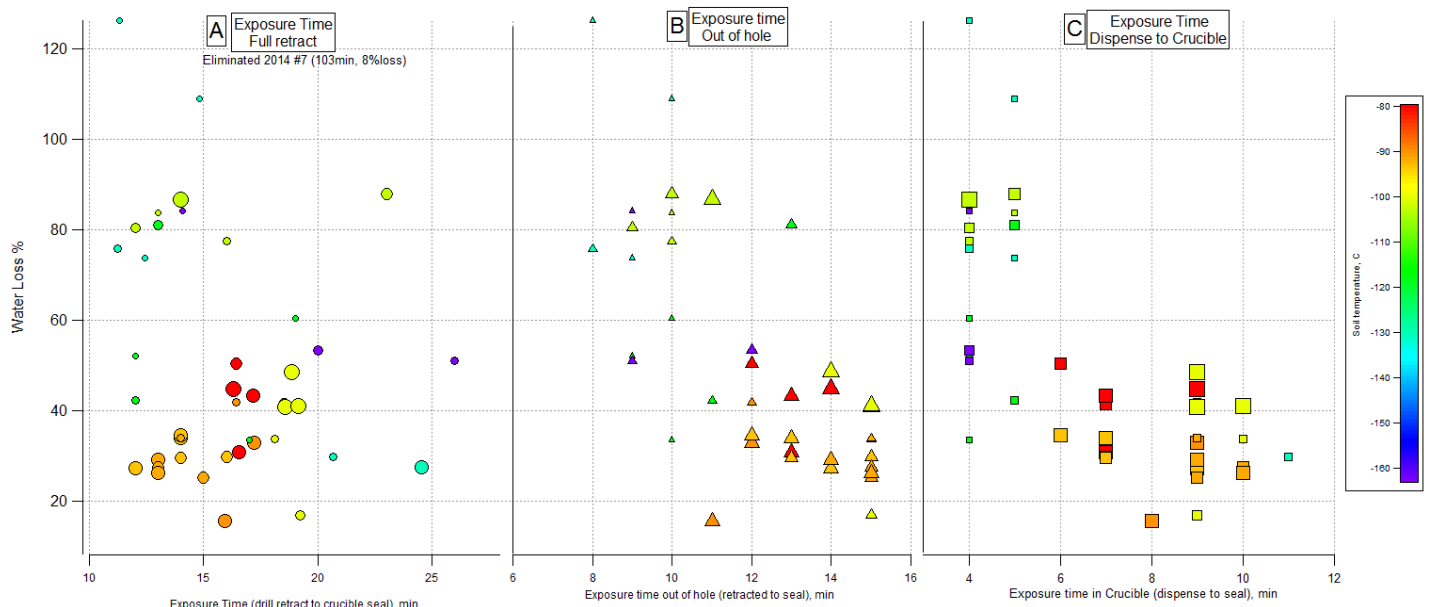

Figure 12. Water loss as a function of exposure time. (A) is time of sample retraction through delivery. $(B)$ is the time the sample is out of the hole, and (C) is delivery (brushing into crucible) only.

Thus far, only 3 samples obtained from the OVEN. While data is limited, thus far the difference between the SCM and OVEN samples in these thermal vacuum chamber tests is not great. The two differences between the OVEN and the SCMs sampling methods are the temperature and the sealing method. All oven crucibles were contained in $20^{\circ} \mathrm{C}$ environment until sample was delivered. While the exact temperature of these crucibles could not be measured, it would be greater than the $-20^{\circ} \mathrm{C} \mathrm{SCM}$ crucible temperatures. Based on post-test analysis, the OVEN crucibles sealed better than the SCMs. Seal integrity was determined by measuring weight gain when the crucibles were opened. The crucibles should gain a few milligrams of mass as atmospheric air fills the vacuum in the crucible. However, neither of these factors appeared to have significant impact on the results. The crucible temperatures indicated in Figure 11D did not show a significant impact. Likewise the OVEN results fell in the same range as the SCM results for the same soil conditions. For a $2.5 \mathrm{wt} \%$ soil bed preparation OVEN losses were between $51 \%$ and $84 \%$, while SCM results ranged $34 \%$ to $81 \%$. The seal integrity measurement had no impact on the spread in the SCM results. 


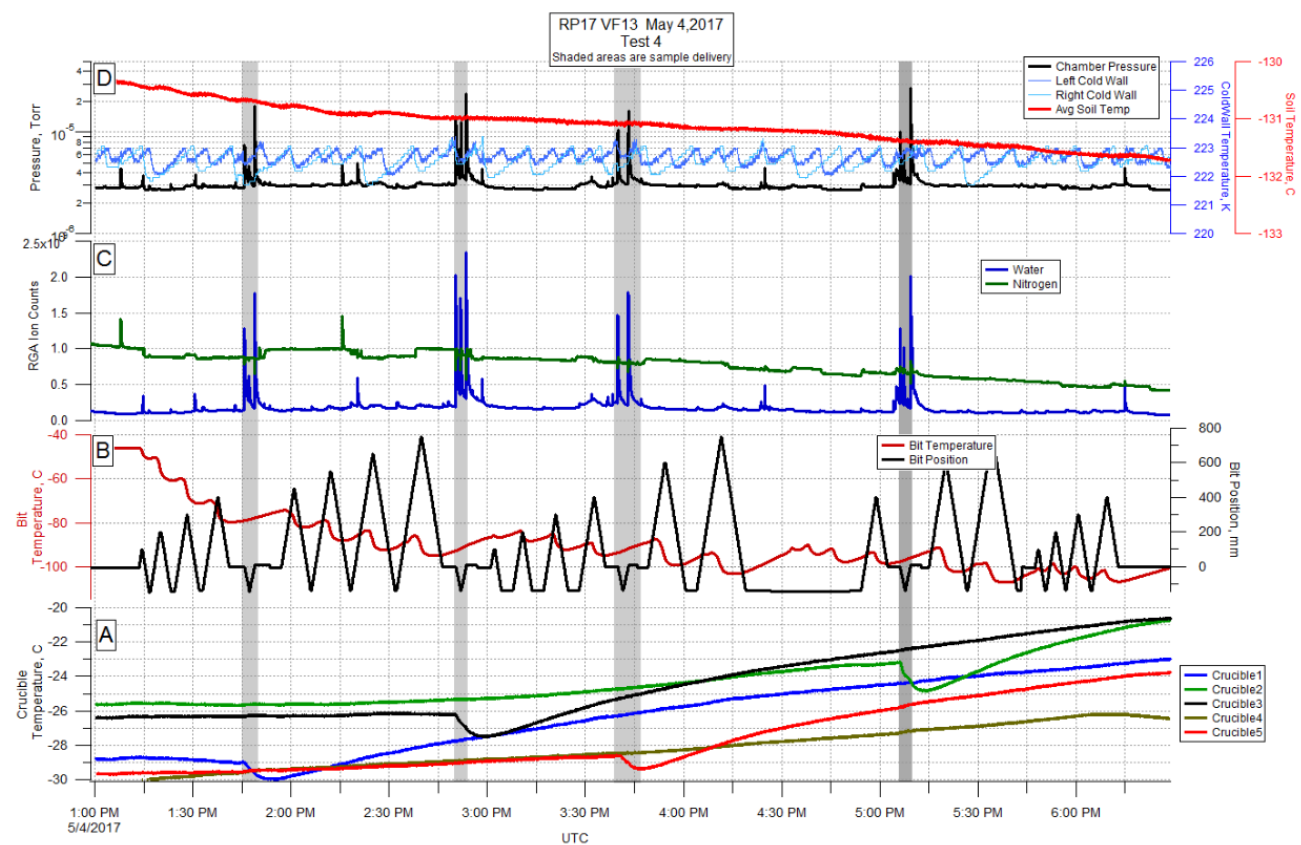

Figure 13. An time lapse overview of one full test encompassing 4 drill holes and 4 samples. (A) is crucible temperature, $(B)$ is the drill position where peaks correspond to depth. (C) is the RGA water signal and (D) shows chamber pressure, temperature, and soil bed temperature.

\section{CONCLUSION}

Resource Prospector development hardware has been tested in a thermal vacuum chamber to examine volatiles loss during subsurface regolith sample acquisition. A total of 43 viable samples have been recovered and analyzed. The testing program has covered a variety of test conditions to address questions regarding mission concept of operations and hardware development. The complexity of the process, in terms of the exposure of the sample to various hardware and environmental conditions, combined with variables of the test program itself has made definitive conclusions difficult. However, current observations suggest the act of delivering the sample into the crucible has a significant impact on the volatiles loss. The agitation of the sample, which exposes a greater amount of regolith surface area, appears to cause a peak in water volatile loss, as evident in the RGA results. The NIRVSS instrument, which looks at the drill cuttings pile during the hole formation, also sees an increase in the presence of water during agitation, eg: when the pile collapses or when the drill percusses.

Mass loss rates of the samples were calculated to take into account exposure time. These rates should be representative of sublimation rate, though at the time of this manuscript a direct correlation is not available. (The complexity of the water/soil mixture, variations in particles size, and test variables necessitate numerical predictions, where analytical correlations such as Andreas, E. (2007) are not adequate). The calculated mass loss rates for the samples are consistent for similar 
sample sizes. Therefore, a sample from wetter soil would retain a greater percentage of water. However, the scatter in the water retention at similar samples size and soil bed conditions is not insignificant. This scatter does not appear to have a clear correlation to exposure temperature nor exposure times that have been measured thus far. Smaller scale laboratory tests are currently being conducted to better understand the mechanical sample delivery process and its impact on the variability in water loss.

\section{ACKNOWLEDGEMENTS}

Resource Prospector is funded by the NASA Advanced Exploration Systems (AES) program.

\section{REFERENCES}

Andreas, E. (2007). "New estimates for the sublimation rate for ice on the Moon". Icarus, Volume 186, Issue 1, January 2007, Pages 24-30

Andrews, D, Coleprete, A., Quinn, J., Chavers, D., and Picard, M. (2014). "Introducing the Resource Prospector (RP) Mission," AIAA Space Conference and Exposition, San Diego, CA., AIAA2014-4378

ASTM (2005). Standard Test Methods for Laboratory Determination of Water (moisture) Content of Soil and Rock by Mass, Standard D2216-05, ASTM International, West Conshohocken, PA.

Colaprete, A., et al, (2010). "Detection of Water in the LCROSS Ejecta Plume", Science, 22, 463-468.

Kleinhenz, J. (2014). "Lunar Polar Environmental Testing: Regolith Simulant Conditioning". AIAA SciTech 2014: 52 ${ }^{\text {st }}$ Aerospace Sciences Meeting and Exhibit. American Institute for Aeronautics and Astronautics. AIAA-20140689

Kleinhenz, J., Paulsen, G., Zacny, K., and Smith, J. (2015). "Impact of Drilling Operations on Lunar Volatiles Capture: Thermal Vacuum Tests". AIAA SciTech 2015: 53 ${ }^{\text {rd }}$ Aerospace Sciences Meeting and Exhibit. American Institute for Aeronautics and Astronautics. AIAA-2015-1177.

Kleinhenz, J.E. and Linne, D., (2013). "Preparation of a Frozen Regolith Simulant Bed for ISRU Component Testing in a Vacuum Chamber", 51st Aerospace Sciences Meeting and Exhibit. American Institute for Aeronautics and Astronautics. AIAA-2013-0732. NASA/TM-2013-217833.

Paulsen, G., Zacny, K., Yaggi, B., Wang, A., Hyman, C., Hedlund, M., Mellerowicz, B., Quinn, J., Smith, J. Development and Testing of the Lunar Resource Prospector Drill (RPD), ASCE Earth and Space Conference, April 11-15, 2016, Orlando, FL

Roush, T. L. et al., (2016) Near-Infrared Monitoring of Volatiles in Frozen Lunar Simulants While Drilling, 9th Symposium on Space Resource Utilization, American Institute for Aeronautics and Astronautics, AIAA 2016-0288, doi:10.2514/6.2016-0228.

Zacny, et al., (2013) Reaching $1 \mathrm{~m}$ deep on Mars: The Icebreaker Drill. Astrobiology 13, DOI: $10.1089 /$ ast.2013.1038. 